\title{
Design and Implement FPGA-Based Hardware and Software for Mobile Bioinformatics
}

\author{
R Shoeibi ${ }^{1, *} ;$ M Mohammadpoor $^{2}$; A Shoeibi Omrani ${ }^{3}$; M Kianmehr ${ }^{4}$ \\ ${ }^{1}$ MSc, Technology Incubator Center Herbal Medicine Medical Equipment, Gonabad University of Medical Sciences, Gonabad, Iran \\ ${ }^{2} \mathrm{PhD}$, Electrical and Computer Department, University of Gonabad, Gonabad, Iran \\ ${ }^{3}$ MSc, Department of Medical Physics, Gonabad University of Medical Sciences, Gonabad, Iran \\ ${ }^{4} \mathrm{PhD}$, Department of Medical Physics, Gonabad University of Medical Sciences, Gonabad, Iran \\ * Corresponding author: R Shoeibi, MSc Graduates, Electronic Engineering, Technology Incubator Center Herbal Medicine Medical Equipment, Gonabad University of Medical Sci- \\ ences, Gonabad. Iran. Tel:+98-9154577991, E-mail: shoeibi@gmu.ac.ir
}

Received: 11 Dec 2016

Accepted: 01 Jan 2017

Epub: 23 Feb 2017

Ppub: 15 Jan 2018

\begin{abstract}
Background:Bioinformatics is producing valuable information using basic biological information such as DNA, RNA sequencing, and proteins by using math and computer science to model biological processes. In the last decades, bioinformatics performed huge projects such as the Genome project, which started in 1991 and ended in 2001. Another important project was coding by collecting common data from functional elements of the human genome, which lasted from 2003 to 2012 using next-generation sequencing technology. Size of biological databases is increasing rapidly. In regards to software-based methods and limitations for biological sequence, there is a need for implementing non-software methods for this purpose. Field-programmable gate arrays (FPGA) has the highest processing speed between hardware equipment and is ideal for accelerating the speed of biological sequences matching. Objectives: The purpose of this paper is design and implementation of hardware for accelerating biological sequence matching and also designing a web-based mobile application to display the information received from the hardware.

Methods: Considering related works, a FPGA-based hardware is designed in this way, which is programmed using very-high-speed integrated circuits hardware description language (VHDL) and is implemented on a FPGA Spartan-3 model using the Xilinx 14.2 software. Also Internet blocks is designed inside the FPGA to send the result of biological sequence matching to the mobile application via Internet. In addition, a software is designed for mobile phones based on the Android operating system to receive the data transmitted by the hardware through the Internet, to show it graphically and numerically, calculate the score matching and identify the genes controversial.

Results: The proposed system is working properly and several times faster than software-based methods. For example, for blood cancer, the chip that is doing gene matching and detecting leukemia.

Conclusion: By this design of hardware implementation, early diagnosis of genetic diseases can be achieved in early stages. For funding the research and production of this technology in the country, advanced drug delivery and gene diagnosis will be achieved. Therefore, health care costs are decreased and treatment is becoming more convenient.

Keywords:Bioinformatics; FPGA; Sequence Matching; Mobile; Speed
\end{abstract}

